\title{
802.16 MCF for 802.11a Based Mesh Networks: A Case for Standards Re-use
}

\author{
Petar Djukic and Shahrokh Valaee \\ The Edward S. Rogers Sr. Department of Electrical and Computer Engineering \\ University of Toronto, 10 King's College Road, Toronto, ON, M5S 3G4, Canada \\ e-mail: djukic,valaee @ comm.utoronto.ca
}

\begin{abstract}
-802.16 and 802.11a have similar physical layer characteristics, however their medium access control is quite different. We present a method to implement 802.16 medium access control with its mesh coordination function (MCF) over existing 802.11a hardware. Our method can be implemented in software, allowing the already deployed 802.11 based mesh nodes to take advantage of the 802.16 MCF, hence increasing the operational lifetime of already installed hardware.

The method works by embedding each 802.16 packet into an 802.11 broadcast packet with some padding, so that the resulting packets can be scheduled with Time Division Multiple Access scheduling. We also show that even after embedding and padding the bandwidth of the resulting system is comparable to the bandwidth that can be achieved with 802.16 hardware. Our method is presented as an interim solution for the upcoming 802.11s standard, as there is currently no hardware working with that standard.
\end{abstract}

Index Terms-Mesh networks, MAC protocol implementation

\section{INTRODUCTION}

Wireless mesh networks provide a cost effective internet connectivity for wireless terminals spread out over a large area. The wireless terminals connect to access points at the edge of the network and the access points are interconnected with wireless links creating a wireless backbone, i.e. the mesh. The nodes in the wireless backbone are called mesh nodes. The wireless backbone forwards packets from the wireless terminals to the base station, and from the base station to the wireless terminals. The base station is the only node in the network connected to public internet, acting as a Point-ofPresence (POP) for the mesh network.

The rationale behind the use of wireless mesh networks is twofold. First, mesh networks can be used to provide firsthop connectivity where it may be expensive or impractical to provide wired first-hop connectivity. Mesh networks provide advantage in this case, since wireless terminals are connected to the mesh network with wireless links, which removes the necessity of having the terminal connected to the backbone with a wire. Second, mesh networks can be used to decrease the cost of providing broadband coverage over a wide geographical area. Mesh networks provide an advantage in this case as well, since mesh nodes are usually designed to use inexpensive off-the-shelf parts such as IEEE 802.11 wireless cards, which use the free radio spectrum.

In line with this rationale, wireless mesh are used to create interconnected wireless hotspots. Wireless hotspots are

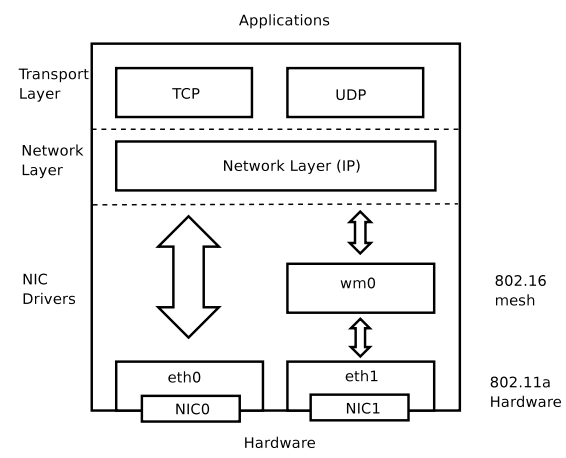

Fig. 1. Modified Network Stack

designed to provide wireless LAN access to authorized mobile terminals such as laptops and PDAs in cafes, airports and malls [1]. Mesh networks can decrease the cost of running wireless hotspots in a common geographical area since they only require a single POP broadband connection to connect all of the hotspots to the internet [2]. For example, using a mesh network to interconnect 133 existing hotspots in the Toronto downtown core could decrease the total cost of running the hotspots by $70 \%$ [3].

Due to their commercial application, it is essential that mesh nodes cooperate in their transmission schedules, so that the utilization of the network is maximized. This is opposite from the view of ad-hoc networks where by definition nodes are designed to rely as little as possible on their neighbors, so coordination is not possible. In the light of the mesh node cooperation it is possible to speak of a mesh coordination function (MCF), which coordinates mesh node transmissions to maximize network utilization. ${ }^{1}$

IEEE has standardized 802.16 based mesh networks standard [5] and is currently working on the $802.11 \mathrm{~s}$ mesh network standard [4]. Both standards use Time Division Multiplexing Access (TDMA) based MAC protocols, with distinct MCFs. In this paper, we argue that the $802.16 \mathrm{MCF}$ can be used in the context of 802.11a based mesh networks ${ }^{2}$ with no hardware changes. This makes it appealing to consider implementing

\footnotetext{
${ }^{1}$ The concept of MCF is proposed in [4] since the normal 802.11 ad-hoc coordination is achieved with the Distributed Coordination Function (DCF).

${ }^{2}$ Many of the current mesh networks implementations are based on 802.11 standard, e.g. [2], [6]-[8]
} 
802.16 over $802.11 \mathrm{a}$ based physical devices until $802.11 \mathrm{~s}$ devices become available and cost effective.

We present a method to embed each 802.16 packet into an 802.11 broadcast packet with some padding, so that the resulting packets can be scheduled with the 802.16 MCF. We show that even after the embedding and the padding, the bandwidth of the resulting system is comparable to the bandwidth that can be achieved with 802.16 hardware.

We are currently working on the implementation of 802.16 MCF over 802.11a hardware devices at the University of Toronto WIRLab. Our implementation only requires the modification of the network stack on the mesh nodes, so it can be used with the hardware on the currently installed mesh networks. We insert an 802.16 mesh driver between the network layer and the 802.11 driver (Fig. 1).

The 802.16 driver packs packets coming from the network layer into 802.16 PDUs. The 802.16 driver then creates 802.11 broadcast packets with the 802.16 PDUs as their contents, which are passed to the 802.11 network interface for transmission. The 802.16 driver adds a custom ethernet type to each packet so that when the packet is received by the peer mesh node it will be transfered to the 802.16 module rather than the network layer.

\section{802.16 Medium ACCESS ConTrol}

IEEE 802.16 is a Time Division Multiple Access (TDMA) based MAC protocol [5]. As any other TDMA MAC, 802.16 builds on a Time Division Multiplexing (TDM) physical layer. In TDM physical layers, the time is divided into time slots of equal length, and during each time slot a block of bytes is broadcast. This division is shown in Fig. 2a. However, in order for TDM based MAC protocols to be implementable, the time slots are grouped into frames of equal length as shown in Fig. 2b. The frames are then repeated over time. IEEE 802.16 MCF specifies how the time slots in each frame are divided among the nodes.

First, we describe the physical layer used in 802.16 and compare it to the physical layer used in 802.11a. 802.16 uses OFDM to implement the TDM physical layer, since all transmissions can be aligned by the length of OFDM symbols. IEEE 802.11a also uses OFDM, but to achieve high data rates.

\section{A. 802.16 Physical Layer}

IEEE 802.16 uses Orthogonal Frequency Division Multiplexing in the license-exempt $5 \mathrm{GHz}$ frequency band. OFDM transforms blocks of bits into constant duration symbols carried on a set of frequency orthogonal pilot carriers. The bandwidth of the final signal is the frequency range occupied by all of the pilot carriers. The duration of each symbol, $T_{\text {Symbol }}$, depends on the bandwidth of each subcarrier, which determines time needed to transmit an unguarded OFDM symbol, $T_{\mathrm{FFT}}$. OFDM also adds a guard time, $T_{\mathrm{G}}$, to each symbol to collect multipath and keep the sub-carriers orthogonal. Both $T_{\mathrm{FFT}}$ and $T_{\mathrm{G}}$ are well known from standard specifications.

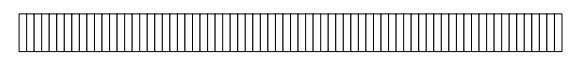

(a) Time division multiplexing with time slots

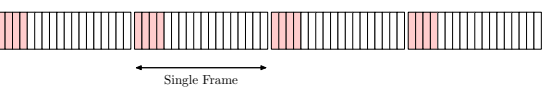

(b) Grouping of time slots into Frames

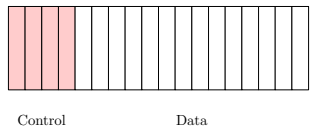

(c) Structure of a Single Frame

Fig. 2. Time Division Multiple Access

TABLE I

COMPARISON OF 802.11A AND 802.16 OFDM

\begin{tabular}{lccc}
\hline Parameter & $\begin{array}{c}802.11 \mathrm{a} \\
(20 \mathrm{MHz})\end{array}$ & $\begin{array}{c}802.16 \\
(10 \mathrm{MHz})\end{array}$ & $\begin{array}{c}802.16 \\
(20 \mathrm{MHz})\end{array}$ \\
\hline Data Carriers & 48 & 192 & 192 \\
Total Carriers & 52 & 256 & 256 \\
$T_{\mathrm{FFT}}$ & $32 \mu \mathrm{s}$ & $22 \frac{2}{9} \mu \mathrm{s}$ & $11 \frac{1}{9} \mu$ \\
$T_{\mathrm{G}}$ & $08 \mu \mathrm{s}$ & $2 \frac{7}{9} \mu \mathrm{s}$ & $1 \frac{7}{18} \mu$ \\
$T_{\text {Symbol }}$ & $4 \mu \mathrm{s}$ & $25 \mu \mathrm{s}$ & $125 \mu \mathrm{s}$ \\
\hline
\end{tabular}

\section{B. 802.16 Frame Structure}

The total length of the 802.16 frame is not mandated by the standard, however there are only a few standard frame lengths. The frame is divided into the control subframe and the data subframe, as is the case in Fig. 2c.

The control subframe is divided into MSH-CTRL-LEN minislots each occupied by a single control packet. Fig. 3 shows a single control transmission and its division into 7 OFDM symbols. Three of the symbols are used to guard the packet, while the other four carry the payload. The packets are modulated with the rate of BPSK-1 2 making the largest possible size for a control packet 48 bytes.

The data subframe is also divided into minislots with a fixed length. The length of data minislot is determined by dividing the number of OFDM symbols in the data subframe with $256 .^{3}$ The timing structure of data packets is similar to the structure of control packets, shown in Fig. 3, except that the data portion is variable. For example, if the minislot size was 4 OFDM symbols the smallest data packet size at BPSK12 modulation, would be 12 bytes, the next size for a data packet is 24 bytes for two minislots, and then 36 bytes for three minislots.

\footnotetext{
${ }^{3}$ For frame lengths less than $8 \mathrm{~ms}$ the minislot size is $T_{\text {Symbol }}$.
} 
TABLE II

COMPARISON OF 802.11A AND 802.16 RAW DATA RATES

\begin{tabular}{|c|c|c|c|c|c|}
\hline \multirow[b]{2}{*}{ Modulation } & \multicolumn{2}{|c|}{ Data $\frac{\text { Bits }}{S \text { Sumbol }}$} & \multicolumn{3}{|c|}{ Bitrate $\frac{\text { Mbits }}{\text { second }}$} \\
\hline & 802.11 & 802.16 & 802.11 & $\begin{array}{l}802.16 \\
10 \mathrm{MHz}\end{array}$ & $\begin{array}{l}802.16 \\
20 \mathrm{MHz}\end{array}$ \\
\hline BPSK-1 2 & 24 & 96 & 6.0 & 3.84 & 7.68 \\
\hline BPSK-3 4 & 36 & $\mathrm{X}$ & 9.0 & $\mathrm{X}$ & X \\
\hline QPSK-1 2 & 48 & 192 & 12.0 & 7.68 & 15.36 \\
\hline QPSK-3 4 & 72 & 288 & 18.0 & 11.52 & 23.04 \\
\hline 16QAM-1 2 & 96 & 384 & 24.0 & 15.36 & 30.72 \\
\hline 16QAM-3 4 & 144 & 576 & 36.0 & 23.04 & 46.08 \\
\hline 64QAM-2 3 & 192 & 768 & 48.0 & 30.72 & 61.44 \\
\hline 64QAM-3 4 & 216 & 864 & 54.0 & 34.56 & 69.12 \\
\hline
\end{tabular}

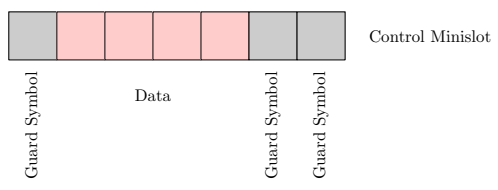

Fig. 3. Structure of 802.16 MAC Control Minislot

\section{802.16 Mesh Coordination Function}

The 802.16 MCF controls the transmission schedules in the control and data subframes. The control subframe is logically represented by a single channel, so a transmission schedule for the control subframe maps minislots to nodes. On the other hand, the data subframe has many logical channels each representing a directional connection between two mesh nodes, so a transmission schedule in the data subframe maps a starting minislot and duration in minislots to a link.

The control subframe transmission scheduling is specified by the standard. However, the standard leaves open how transmission schedules in the data subframe are determined and only specifies the mechanisms by which transmission schedules in the data subframe are announced. The 802.16 MCF specifies two kinds of scheduling protocols:

1) Centralized Scheduling Protocol: The base station decides transmission schedules for all links in the network. The schedule is flooded through the network, so that all mesh nodes know the entire transmission schedule.

2) Decentralized Scheduling: The nodes negotiate the starting minislot and duration for each link. The protocol uses handshakes to ensure all neighbouring nodes are aware of the transmission schedules.

\section{EMBEDDED 802.16 MCF OVER 802.11 HARDWARE}

We propose embedding 802.16 MAC packet data units (PDUs) into 802.11a MAC broadcast packets. The RTSCTS-ACK exchanges are not necessary since 802.16 MCF coordinates the nodes, and acknowledgments are a part of the 802.16 protocol.

Fig. 4 shows the embedding of the 802.16 packets into the

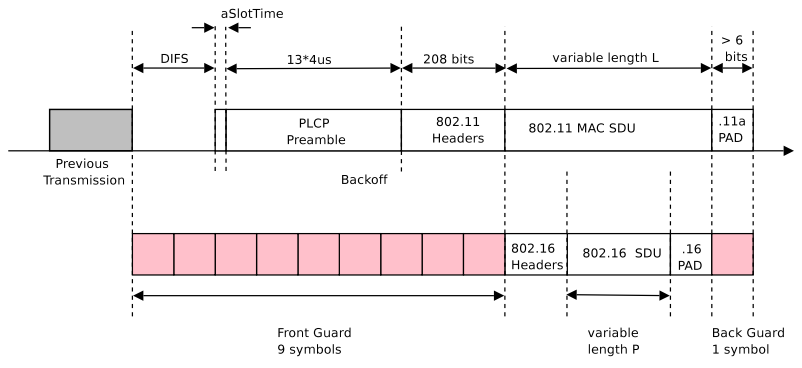

Fig. 4. Embedding 802.16 PDU over 802.11a

802.11a broadcast packets. ${ }^{4}$ The embedding assumes that the back-off time is always one. For this to be true, we need to set CWmin $=$ CWmax $=1 .^{5}$ The length of the PLCP preamble includes 12 training symbols as well as one OFDM symbol that carries the PLCP header, for a total of 13 symbols [11]. 802.11 headers include 2 bytes for the symbol field (specific to 802.11a), as well as 24 bytes for 802.11 MAC headers [11], [12].

The transmission time required to transfer $L$ bits of the 802.16 packet is given by:

$$
T_{\text {tran }}^{80211}=95 \mu \mathrm{s}+\left\lceil\frac{214+L}{r_{\bmod }^{80211}}\right\rceil \times 4 \mu \mathrm{s}
$$

where $r_{\text {mod }}^{802} 11$ is the number of data bits in an 802.11 OFDM symbol (Table II).

The bottom part of Fig. 4 shows how 802.16 MAC interprets the embedded transmission. We set the minislot duration $T_{\mathrm{MS}}=16 \mu \mathrm{s}$, so each minislot corresponds to four $802.11 \mathrm{a}$ OFDM symbols. ${ }^{6}$ We modify the settings for the 802.16 MAC protocol to make the front guard time 9 minislots and the back guard time one minislot (Fig. 3). We chose the value of 9 for the front guard because that is longer than the time required to transmit all of 802.11a overhead with any modulation rate. The back guard symbols are the minimum required to transmit 802.11a padding.

IEEE 802.16 standard calculates the transmission time as:

$$
T_{\text {tran }}^{80216}=\left[\left\lceil\frac{P}{r_{\bmod }^{802} 16}\right\rceil+10\right] \times 16 \mu \mathrm{s}>T_{\text {tran }}^{80211}
$$

where $r_{\text {mod }}^{802} 16$ is the number of data bits in an 802.16 symbol (Table II). The transmission time seen by 802.16 MAC is strictly greater than the time actually required to transmit the packet over 802.11a, making collisions impossible.

\section{A. Performance of the embedded 802.16}

Fig. 5 and Fig. 6 compare the performance of the embedded transmissions to the performance of 802.16. We used (2)

\footnotetext{
${ }^{4}$ It is possible to embed the packets in conjution with the sub-network access protocol (SNAP) [9]. However, this is not necessary under Linux because the kernel allows the definition of custom ethernet types, so we opted to use an embedding with a smaller size, i.e. without the SNAP sub-header.

${ }^{5}$ For real implementations, this depends on support by the firmware. For example, the Intel PRO/Wireless 2900bg cards do allow this change [10].

${ }^{6}$ This minislot duration maps one 802.16 OFDM symbol to $T_{\mathrm{MS}}$ (Table I).
} 


\section{3rd Biennial Symposium on Communications}

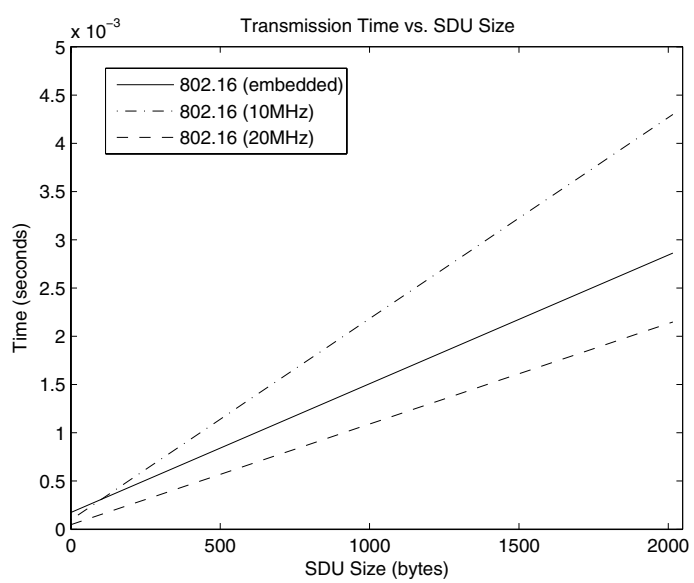

Fig. 5. Transmission Time Comparison (BPSK-1/2)

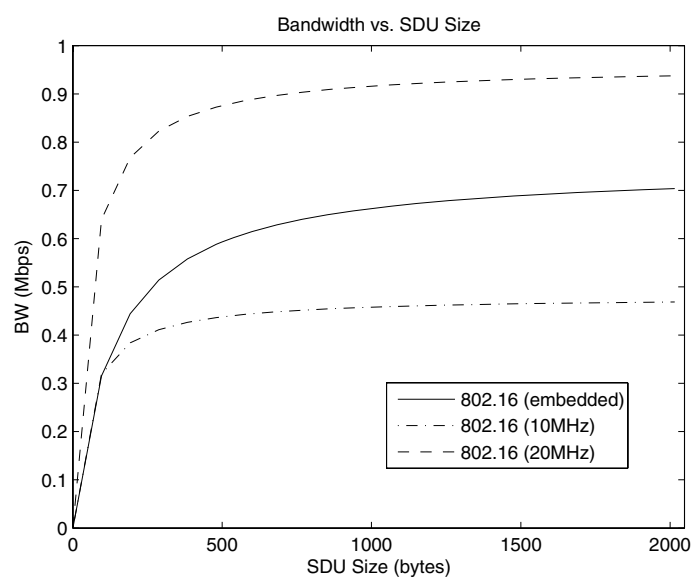

Fig. 6. Bandwidth Comparison (BPSK-1/2)

to calculate the transmission times of the embedded 802.16. Since 802.16 uses a collision free MCF we do not include any backoffs in the two figures.

Fig. 5 shows the transmission times required to transfer a single SDU. We observe for small SDU sizes the transmission time of the embedded 802.16 can be larger than the transmission time of 802.16 hardware operating at $10 \mathrm{MHz}$. For all other SDU sizes the transmission times of the embedded 802.16 are always smaller than the transmission times of 802.16 hardware operating at $10 \mathrm{MHz}$, and more than the transmission times of 802.16 hardware operating at $20 \mathrm{MHz}$. This corresponds to the fact that the actual symbol duration in the embedded scheme is $16 \mu \mathrm{s}$, compared to $125 \mu \mathrm{s}$ and $25 \mu \mathrm{s}$ for 802.16 hardware operating at $20 \mathrm{MHz}$ and $10 \mathrm{MHz}$ respectivelly (Table I).

Fig. 6 shows the achievable bandwidth when the modulation is BPSK-1 2. The achievable bandwidth is calculated by dividing the SDU size with the transmission time. First, we observe that the achievable bandwidth of the embedded scheme is comparable to the bandwidth that can be achieved using 802.16 hardware. The achievable bandwidth of the embedded scheme is less than what can be achieved with 802.16 hardware operating at $20 \mathrm{MHz}$, and more than the bandwidth of 802.16 hardware operating at $10 \mathrm{MHz}$.

\section{SUMmARY AND FUTURE WORK}

We have presented a method that allows $802.16 \mathrm{MCF}$ to be used for synchronization of data transmissions in $802.11 \mathrm{a}$ mesh networks. The method works by embedding 802.16 packets into $802.11 \mathrm{a}$ broadcast packets with padding so that all transmissions can be aligned on 802.16 frame boundaries. Our method adds little overhead to 801.11a broadcasts and actually decreases the overhead inhearent in RTS-CTS-ACK exchanges which are a part of the 802.11 DCF. At the same time our method removes collisions from the channel because the nodes are communicating in 802.16 TDMA fashion.

Our method requires only software changes on the nodes which are using 802.11a for mesh communications. This means that the mesh networks installed with 802.11a hardware today can be upgraded with a software patch to take advantage of 802.16 MCF and do not have to wait for hardware upgrades to $802.11 \mathrm{~s}$.

\section{REFERENCES}

[1] P. Bahl, W. Russell, Y.-M. Wang, A. Balachandran, G. M. Voelker, and A. Miu, "PAWNs: Satisfying the need for ubiquitos secure connectivity and location services," IEEE Wireless Commun. Mag., vol. 9, no. 1, pp. 84-89, February 2002.

[2] Nortel Networks, "Wireless mesh network - extending the reach of wireless lan, securely and cost-effectively," http://www.nortelnetworks.com/solutions/wlan/, November, 2003.

[3] M. Chee, "The business case for wireless mesh networks," December 2003.

[4] IEEE, "802.11 TGs MAC enhacement proposal," IEEE, Protocol Proposal IEEE 802.11-05/0575r3, September 2005.

[5] "IEEE standard for local and metropolitan area networks part 16: Air interface for fixed broadband wireless access systems," 2004.

[6] B. A. Chambers, "The grid roofnet: a rooftop ad hoc wireless network," M. Eng., Massachusetts Institute of Technology, 2002.

[7] A. Adya, P. Bahl, J. Padhye, A. Wolman, and L. Zhou, "A multi-radio unification protocol for IEEE 802.11 wireless networks," in BroadNets, 2004.

[8] J. Camp, J. Robinson, C. Steger, and E. Knightly, "Measurement driven deployment of a two-tier urban mesh access network," Rice University, Technical Report TREE0505, December 2005.

[9] "IEEE standard for local and metropolitan area networks: Overview and architecture," 2001.

[10] "Intel ${ }^{\circledR}$ PRO/Wireless 2200BG driver for linux," http://ipw2200. sourceforge.net/.

[11] "IEEE standard for local and metropolitan area networks part 11: Part 11: Wireless lan medium access control (MAC) and physical layer (PHY) specifications high-speed physical layer in the 5 ghz band," 1999.

[12] "IEEE standard for local and metropolitan area networks part 11: Wireless lan medium access control (MAC) and physical layer (PHY) specifications," 1999. 